\title{
AN INSTABILITY THEOREM FOR A CERTAIN SIXTH ORDER DIFFERENTIAL EQUATION
}

\author{
J. O. C. EZEILO \\ (Received 6 June 1979; revised 3 February 1981) \\ Communicated by G. R. Morris
}

\begin{abstract}
The object of the paper is to establish an instability theorem for sixth order differential equations of the form (1.1). The proof is based on the use of Krasovskii criteria.
\end{abstract}

1980 Mathematics subject classification (Amer. Math. Soc.): 34 D 99.

1.

We shall be concerned here with the instability of the trivial solution $x=0$ of differential equations of the form:

$$
\begin{aligned}
& x^{(6)}+a_{1} x^{(5)}+a_{2} x^{(4)}+\theta\left(x, \dot{x}, \ddot{x}, \ddot{x}, x^{(4)}, x^{(5)}\right) \ddot{x} \\
& +f(\dot{x}) \ddot{x}+g\left(x, \dot{x}, \ddot{x}, \ddot{x}, x^{(4)}, x^{(5)}\right) \dot{x}+h(x)=0 \quad(h(0)=0),
\end{aligned}
$$

in which $a_{1}, a_{2}$ are constants and $e, f, g, h$ are continuous functions dependent only on the arguments shown.

For the strictly constant-coefficient case:

$$
x^{(6)}+a_{1} x^{(5)}+a_{2} x^{(4)}+a_{3} \ddot{x}+a_{4} \ddot{x}+a_{5} \dot{x}+a_{6} x=0,
$$

a necessary and sufficient condition for the instability of the trivial solution is that the associated auxiliary equation

$$
\psi(r) \equiv r^{6}+a_{1} r^{5}+a_{2} r^{4}+a_{3} r^{3}+a_{4} r^{2}+a_{5} r_{6}=0
$$

has at least one root with a positive real part. This requirement on the roots of (1.3) places a variety of conditions on $a_{1}, \ldots, a_{6}$ some of which can be

(1) Copyright Australian Mathematical Society 1982 
extracted explicitly from purely algebraic considerations in much the same way as was done in Ezeilo (1978) for a corresponding fifth order differential equation. For example, we know from the general theory that (1.3) necessarily has a root $r=\alpha+i \beta$ ( $\alpha, \beta$ real) with $\alpha \geqslant 0$ if one, at least, of the Routh's test determinants $\Delta_{1}, \ldots, \Delta_{6}$ (or orders $1, \ldots, 6$ respectively) is non-positive. By actually multiplying out the determinants concerned, we find that

$$
\begin{aligned}
& \Delta_{4}=-a_{5}^{2}+K_{1} a_{5}+K_{2} a_{6}+K_{3}, \\
& \Delta_{5}=-a_{1}^{3} a_{6}^{2}-a_{5}^{3}+K_{4} a_{5}^{2}+K_{5} a_{5} a_{6}+K_{6} a_{5}+K_{7} a_{6},
\end{aligned}
$$

where $K_{1}, \ldots, K_{7}$ are terms which depend only on $a_{1}, a_{2}, a_{3}$ and $a_{4}$. It is clear from the form of $\Delta_{5}$ that

$$
\Delta_{5} \leqslant 0 \text { if } a_{1}>0, a_{5}>0 \text { and }\left|a_{6}\right| \geqslant K_{8} a_{5}+K_{9}
$$

for some sufficiently large $K_{8}=K_{8}\left(a_{1}, a_{2}, a_{3}, a_{4}\right)>0$ and $K_{9}=K_{9}\left(a_{1}, a_{2}, a_{3}, a_{4}\right)$ $>0$, and from the form of $\Delta_{4}$ that $\Delta_{4}<0$ if $\left|a_{6}\right|<K_{8} a_{5}+K_{9}$ provided that $a_{5}>K_{10}$ for some sufficiently large $K_{10}=K_{10}\left(a_{1}, a_{2}, a_{3}, a_{4}\right)>0$. These show that one or other of $\Delta_{4} \leqslant 0$ or $\Delta_{5} \leqslant 0$ must hold, and therefore that (1.3) has at least one $r_{0}=\alpha_{0}+i \beta_{0}\left(\alpha_{0}, \beta_{0}\right.$ real), with $\alpha_{0}>0$ or $\alpha_{0}=0$, if

$$
a_{1}>0 \text { and } a_{5} \geqslant K_{10} \text {. }
$$

We can obviously rule out the possibility $\alpha_{0}=0$ here if, for example, $\psi\left(i \beta_{0}\right)$ has a non-vanishing imaginary part, that is

$$
i \beta_{0} \theta\left(\beta_{0}\right) \neq 0
$$

where

$$
\begin{aligned}
\theta\left(\beta_{0}\right) & \equiv a_{1} \beta_{0}^{4}-a_{3} \beta_{0}^{2}+a_{5} \\
& =a_{1}\left(\beta_{0}-\frac{1}{2} a_{3} a_{1}^{-1}\right)^{2}+a_{5}-\frac{1}{4} a_{3}^{2} a_{1}^{-1} .
\end{aligned}
$$

The recasting of $\theta\left(\beta_{0}\right)$ in the latter form is possible since $a_{1} \neq 0$, and it shows for instance that (1.5) holds if $\beta_{0} \neq 0$ and

$$
a_{1}>0, \quad a_{5}>\frac{1}{4} a_{3}^{2} a_{1}^{-1} .
$$

Hence we have, on gathering results, that (1.3) has a root $r_{0}=\alpha_{0}+i \beta_{0}$ with $\alpha_{0}>0$, and therefore we expect instability for the trivial solution of (1.2) with $a_{6} \neq 0$, if

$$
a_{1}>0 \text { and } a_{5}>\max \left(K_{10}, \frac{1}{4} a_{3}^{2} a_{1}^{-1}\right) .
$$

The above (somewhat lengthy) preamble is merely intended to give the background to the hypotheses which play a dominant role in our treatment here and to give some indication of how these hypotheses stand with respect to the Routh-Hurwitz criteria. Indeed we found that, by using the well known criteria of Krasovskii (1955) it is possible not only to arrive at the same instability results 
for (1.2) much more briskly, and with an improved restriction on $a_{5}$, but also to extend results to the more general equation (1.1). We shall in fact prove that

THEOREM. Given the equation (1.1) in which $a_{1} \neq 0$ suppose that

$$
h(x) \neq 0 \text { for } x \neq 0 \text {, }
$$

and that further

$$
g\left(x_{1}, x_{2}, x_{3}, x_{4}, x_{5}, x_{6}\right) \operatorname{sgn} a_{1}>\frac{1}{4}\left|a_{1}\right|^{-1} e^{2}\left(x_{1}, x_{2}, x_{3}, x_{4}, x_{5}, x_{6}\right)
$$

for arbitrary $x_{1}, \ldots, x_{6}$.

Then the trivial solution $x=0$ of $(1.1)$ is unstable.

Observe that, when specialized to (1.2) with $a_{1}>0$ (1.8) reduces to: $a_{5}$ $>\frac{1}{4} a_{3}^{2} a_{1}^{-1}$ which is, in general, sharper than (1.6).

Observe also that (1.7) not only effectively generalizes the condition $a_{6} \neq 0$ for (1.2) but also holds good for functions $h(x)$ such as $\pm|x|, \pm x^{2}$ which do not change signs with $x$.

The theorem as stated above includes the case $a_{1}<0$ not covered in our introductory remarks to the theorem. It is easy to see, however, from the fact that the sum of the roots of (1.3) equals $\left(-a_{1}\right)$, that the two conditions: $a_{1}<0$ and $a_{6} \neq 0$ alone suffice for the instability of the trivial solution of (1.2) so that the theorem, because of its dependence on (1.8), is only a partial generalization of this special case $\left(a_{1}<0\right)$ of $(1.2)$.

\section{Proof of the theorem}

It is convenient to take up (1.1) in the system-form:

(2.1)

$$
\begin{aligned}
& \left\{\begin{array}{l}
\dot{x}_{1}=x_{i+1} \quad(i=1, \ldots, 5) \\
\dot{x}_{6}=-a_{1} x_{6}-a_{2} x_{5}-e\left(x_{1}, \ldots, x_{6}\right) x_{4}-f\left(x_{2}\right) x_{3}-g\left(x_{1}, \ldots, x_{6}\right) x_{2}-h\left(x_{1}\right)
\end{array}\right. \\
& \text { obtained as usual by setting } x_{1}=x, x_{2}=\dot{x}, x_{3}=\ddot{x}, x_{4}=\ddot{x}, x_{5}=x^{(4)} \text { and } \\
& x_{6}=x^{(5)} \text { in (1.1). }
\end{aligned}
$$

Consider the function $U=U\left(x_{1}, \ldots, x_{6}\right)$ defined by

$$
\left\{\begin{aligned}
U= & -x_{2}\left(x_{6}+a_{1} x_{5}+a_{2} x_{4}\right)+\frac{1}{2} a_{2} x_{3}^{2} \\
& +x_{3}\left(x_{5}+a_{1} x_{4}\right)-\frac{1}{2} x_{4}^{2}-\int_{0}^{x_{2}} y f(y) d y-\int_{0}^{x_{1}} h(s) d s .
\end{aligned}\right.
$$


We shall show that the function $V=V\left(x_{1}, \ldots, x_{6}\right)$ given by

$$
V=U \operatorname{sgn} a_{1}
$$

satisfies the six-dimensional version of the (Krasovskii) properties which we had labelled $\left(\mathrm{P}_{1}\right),\left(\mathrm{P}_{2}\right)$ and $\left(\mathrm{P}_{3}\right)$ in Ezeilo (1978), page 346 , if $a_{1} \neq 0$ and the conditions (1.7) and (1.8) of the theorem hold, as we assume henceforth.

First if $k \equiv 1+\left|a_{2}\right|$ it is evident from the definition that

$$
V\left(0,0, \varepsilon \operatorname{sgn} a_{1}, 0, k \varepsilon, 0\right)=\varepsilon^{2}\left(k+\frac{1}{2} a_{2} \operatorname{sgn} a_{1}\right)
$$

for arbitrary $\varepsilon>0$, so that every neighborhood of the origin in the $\left(x_{1}, \ldots, x_{6}\right)$-space contains points $\left(\xi_{1}, \ldots, \xi_{6}\right)$ such that $V\left(\xi_{1}, \ldots, \xi_{6}\right)>0$. Next let

$$
\left(x_{1}, \ldots, x_{6}\right)=\left(x_{1}(t), \ldots, x_{6}(t)\right)
$$

be an arbitrary solution of (2.1). An elementary differentiation will show that

$$
\begin{aligned}
\dot{V} & \equiv \frac{d}{d t} V\left(x_{1}, \ldots, x_{6}\right) \\
& =\left\{a_{1} x_{4}^{2}+e\left(x_{1}, \ldots, x_{6}\right) x_{2} x_{4}+g\left(x_{1}, \ldots, x_{6}\right) x_{2}^{2}\right\} \operatorname{sgn} a_{1} \\
& \equiv\left|a_{1}\right|\left(x_{4}+\frac{1}{2}\left|a_{1}\right|^{-1} e x_{2}\right)^{2}+\left\{g \operatorname{sgn} a_{1}-\frac{1}{4}\left|a_{1}\right|^{-1} e^{2}\right\} x_{2}^{2} .
\end{aligned}
$$

Thus, by (1.8), $\dot{V}$ is positive semi-definite. Also $\dot{V}=0(t>0)$ necessarily implies that

$$
x_{2}=0 \quad(t \geqslant 0)
$$

which in turn implies that

$$
\begin{gathered}
x_{1}=\xi_{1}(\text { constant }), \quad x_{3}=\dot{x}_{2}=0, \quad x_{4}=\ddot{x}_{2}=0, \\
x_{5}=\ddot{x}_{2}=0, \quad x_{6}=x_{2}^{(4)}=0, \quad(t>0) .
\end{gathered}
$$

The substitution of (2.4) and (2.5) in (2.1) leads to the result $h\left(\xi_{1}\right)=0$ which by (1.7) implies (only) that $\xi_{1}=0$. Hence $\dot{V}=0(t>0)$ implies that

$$
x_{1}=0=x_{2}=x_{3}=x_{4}=x_{5}=x_{6} \quad(t>0) .
$$

The function $V$ thus has all the requisite Krasovskii properties subject to the conditions in the theorem, which now follows.

It has not been possible to extend the present methods to the case $a_{1}=0$ (as in (Ezeilo (1978))) or indeed, by considering the real, rather than the imaginary, 
part of $\psi\left(i \beta_{0}\right)$ (refer back here to Section 1), to extract suitable conditions on $a_{2}$, $a_{4}$ and $a_{6}$ which can be utilized in establishing some other instability theorem(s) for an equation (1.2) in which $a_{1}, \ldots, a_{6}$ are not all constants.

\section{References}

J. O. C. Ezeilo (1978), 'Instability theorems for certain fifth order differential equations,' Math. Proc. Cambridge Philos. Soc. 84, 343-350.

N. N. Krasovskii (1955), "Conditions for the converse of Lyapunov theorems on instability for stationary systems of differential equations' Dokl. Akad. Nauk SSSR 101, 17-20.

Univeristy of Nigeria

Nsukka

Nigeria 